\title{
Tomato endophytic bacteria bioactive compounds as potential agents for the postharvest biocontrol of gray mold disease
}

Chaouachi M. ${ }^{1,2,3}$, Marzouk T. ${ }^{1,2}$, Jallouli S. ${ }^{2}$, Elkahoui S. ${ }^{2,4}$, Gentzbittel L. ${ }^{3,5}$, Djébali N. ${ }^{2}$, Ben C. ${ }^{3,5 *}$

${ }^{1}$ Univ. of Tunis El Manar, Tunis, Tunisia

${ }^{2}$ Lab. of Bioactive Substances, Centre of Biotech. of Borj Cedria, Tunisia

${ }^{3}$ Lab. of Functional Ecology \& Environment, CNRS, Univ. of Toulouse, France

${ }^{4}$ Dept of Biol., College of Sciences, Univ. of Ha'il, Saudi Arabia

${ }^{5}$ Digital Agriculture Lab., Skolkovo Institute of Science and Technology, Moscow Region, Russian

Federation

*email:C.Ben@skoltech.ru

Botrytis cinerea is one of the most harmful postharvest fungal pathogens causing significant losses in fresh fruits and vegetables. Safer alternatives to hazardous synthetic fungicides are sought to control postharvest decay loss. The use of antagonistic microorganisms is increasingly popular worldwide. We investigated the antifungal and plant growth promoting activities and the identity of volatile organic compounds (VOCs) produced by tomato-derived endophytic bacteria strains. The capacity of selected strains to prevent postharvest $B$. cinerea infection on tomatoes through VOCs and soluble compounds was studied. A collection of 50 endophytic bacterial strains was established from various organs of tomato plants from Cape Bon (Tunisia). Endophytic bacteria from tomato healthy plants mainly colonize leaves and are significantly enriched in Bacillus strains. In vitro assays showed that $36 \%$ of the strains produce antifungal VOCs against $B$. cinerea and about $14 \%$ produce VOCs with specific promoting effects on tomato seedling length or biomass production. Five strains with fungal antagonistic effect were selected for further analysis. They produced a core set of 7 VOCs along with different strain-specific and known antifungal VOCs. Bio-protection assay on tomato fruits showed that the Enterobacter strain TR1 produces the most protective VOCs against B. cinerea infection with 3-Methylbutan-1-ol as a major volatile compound which totally suppressed $B$. cinerea growth. In contrast, the Bacillus strains showed better protection against fungal infection when applied as vegetative cells. These results support the use of the selected endophytic bacteria strains as potential biocontrol agents to reduce postharvest decay of $B$. cinerea, as well as 3-Methylbutan-1-ol as promising antifungal volatile to apply during postharvest commercialization of tomatoes. 